\title{
Abnormal Erythroid Cell Proliferation and Myelofibrosis in a Cat
}

\author{
Tomoko IWANAGA ${ }^{1)}$, Naoki MIURA ${ }^{1)}$, Noriaki MIYOSHI'), Yasuyuki ENDO ${ }^{3)}$ and Yasuyuki MOMOI ${ }^{1) *}$ \\ 1) Laboratory of Veterinary Diagnostic Imaging, Department of Veterinary Medicine, Joint Faculty of Veterinary Medicine, Kagoshima \\ University, 1-21-24 Korimoto, Kagoshima 890-0065, Japan \\ ${ }^{2)}$ Laboratory of Veterinary Histopathology, Department of Veterinary Medicine, Joint Faculty of Veterinary Medicine, Kagoshima \\ University, 1-21-24 Korimoto, Kagoshima 890-0065, Japan \\ 3) Laboratory of Small Animal Internal Medicine, Department of Veterinary Medicine, Joint Faculty of Veterinary Medicine, \\ Kagoshima University, 1-21-24 Korimoto, Kagoshima 890-0065, Japan
}

(Received 5 June 2011/Accepted 24 January 2012/Published online in J-STAGE 7 February 2012)

ABSTRACT. A cat was presented with severe progressive anemia despite marked erythroblastosis. The cat was negative for feline leukemia virus antigen and feline immunodeficiency virus antibody. Bone marrow cytology revealed an excess of erythroid cells with a predominance of prorubricytes and basophilic rubricytes. No response to immunosuppressive therapy was obtained, and a tentative diagnosis of myelodysplastic syndrome was made. The cat showed a partial response to low-dose cytarabine $\left(20 \mathrm{mg} / \mathrm{m}^{2} \mathrm{subcutane}-\right.$ ously q24) but died 51 days after the 1st admission. Histopathological examination revealed fibrosis in the bone marrow and marked infiltration of erythroid cells into other organs.

KEY WORDS: anemia, feline, myelofibrosis.

doi: 10.1292/jvms.11-0268; J. Vet. Med. Sci. 74(7): 909-912, 2012

Myelofibrosis is a myeloproliferative condition that occurs in conjunction with other myeloproliferative diseases such as leukemia or myelodysplastic syndrome (MDS). Most cats with a myeloproliferative disease are infected with feline leukemia virus (FeLV), and abnormal proliferation of FeLV-infected progenitor cells is believed to be associated with the etiology of the disease [9]. In this report, a cat negative for FeLV infection was diagnosed as having myelofibrosis with marked erythroid proliferation.

An approximately 7-year-old spayed domestic shorthaired cat was referred to Kagoshima University Veterinary Teaching Hospital (KUVTH) with anorexia, pyrexia and severe anemia. The cat had not been vaccinated and had been allowed outdoors. Before admission to KUVTH, the cat had been treated with clindamycin and a blood transfusion but responded poorly to the therapy. At the 1 st examination, the cat had a fever $\left(39.7^{\circ} \mathrm{C}\right)$ and pale mucous membranes. Splenomegaly was apparent by abdominal palpation. A cardiac murmur was auscultated at the apex area (Levine I/VI). Thoracic radiography and ultrasonography revealed an enlarged sternal lymph node and a small amount of pleural effusion. Marked hepatomegaly and splenomegaly with homogeneous echogenicity were also observed on abdominal ultrasonography. Blood examination revealed mild leukocytosis $(23,000 / \mu l)$, severe anemia $\left(\mathrm{RBC}, 198 \times 10^{4}\right.$ / $\mu l$; HT, 9\%; HGB, $3.1 \mathrm{~g} / \mathrm{d} l$ ), moderate thrombocytopenia

\footnotetext{
*Correspondence to: Momol, Y., Laboratory of Veterinary Diagnostic Imaging, Department of Veterinary Medicine, Joint Faculty of Agriculture, Kagoshima University, 1-21-24 Korimoto, Kagoshima 890-0065, Japan.

e-mail:momoi@agri.kagoshima-u.ac.jp

(C)2012 The Japanese Society of Veterinary Science
}

$\left(8.0 \times 10^{4} / \mu l\right)$, elevated lactate dehydrogenase activity (448 $\mathrm{U} / l)$ and decreased serum iron concentration $(32 \mu \mathrm{g} / \mathrm{d} l)$. Other serum chemistry parameters, including ALT, ALP, BUN, creatinine and calcium, were within normal ranges. Serum anti-feline immunodeficiency virus antibody (FIV) and FeLV antigen tests were negative. FeLV provirus was not detected by polymerase chain reaction (PCR) in blood [6]. A number of prorubricytes and basophilic rubricytes appeared in peripheral blood (8\%) with polychromatophilic rubricytes and metarubricytes (16\%) (Fig.1A). Although the numbers of aggregated and punctate reticulocytes were relatively low $(28,980 / \mu l, 0.9 \%)$ (reference ranges: aggregate reticulocytes, $<0.5 \%$; punctate reticulocytes, $<10 \%$ ) [2], we regarded this condition as regenerative anemia at the 1 st examination because a number of erythroid progenitor cells appeared in peripheral blood. Thus, doxycycline (6.7 $\mathrm{mg} / \mathrm{kg}$ q12h) with prednisolone $(2 \mathrm{mg} / \mathrm{kg}$ q12h) was started as an initial treatment with supportive therapy until hemotropic mycoplasma infection was ruled out by PCR [11]. The cat did not respond to this treatment; therefore, bone marrow aspiration and spleen aspiration biopsies were performed 8 days after the 1 st examination. The bone marrow was hypocellular with an excess of erythroid cells (M/E ratio $=0.31)$, prorubricytes $(24.5 \% / \mathrm{ANC})$ and basophilic rubricytes (29.0\%/ANC) (Fig. 1B), but polychromatophilic rubricytes (12.6\%/ANC) and metarubricytes (9.9\%/ANC) were within their normal ranges. Morphological dysplasia of bone marrow cells was minimal and a small subset of erythroid cells showed dysplastic features such as fragmentation of the nucleus and irregular nucleus borders (Fig. 1C). In the spleen, large numbers of prorubricytes and basophilic rubricytes were also identified. Neither mast cells nor other tumor cell types were detected. These findings indicated 

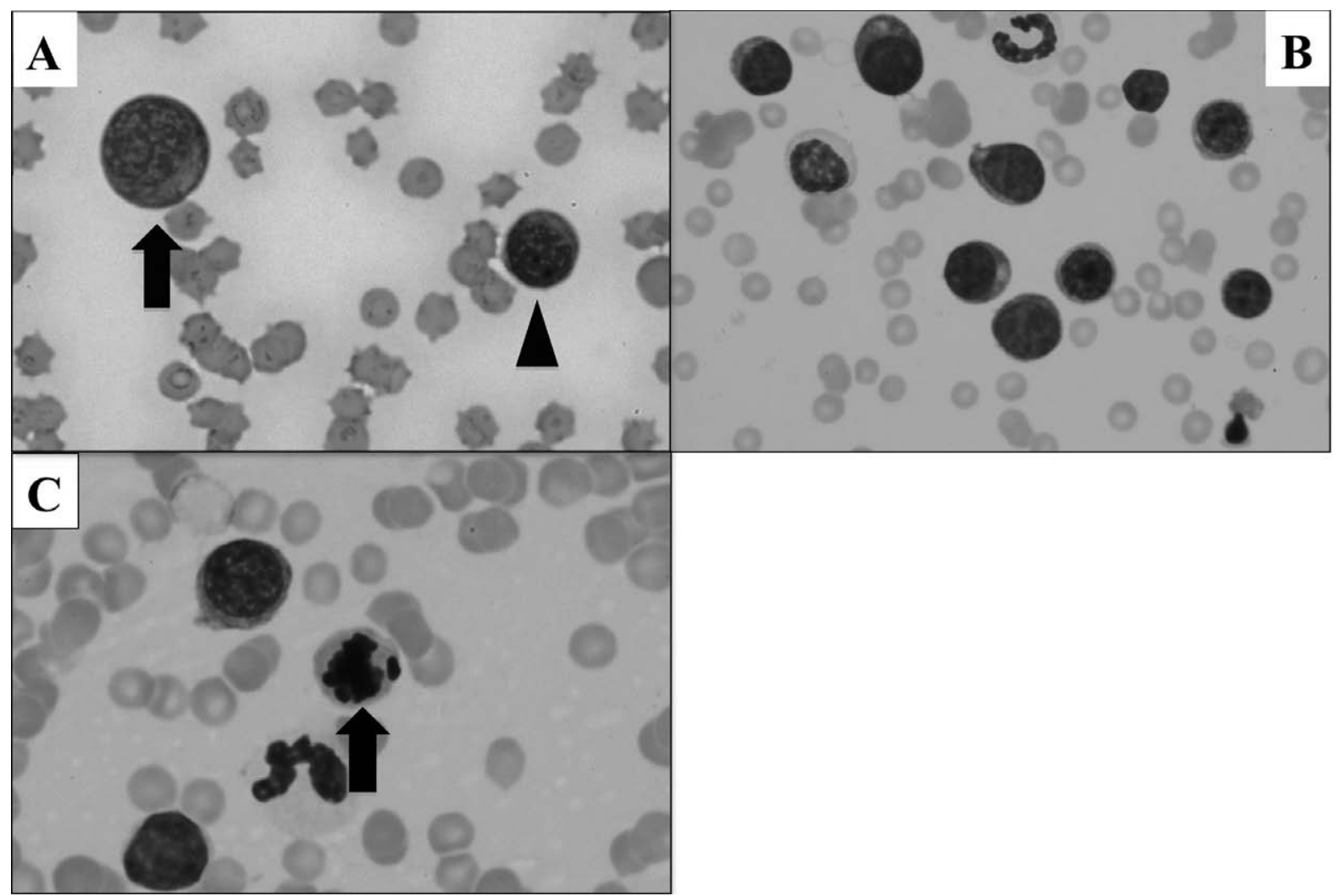

Fig. 1. (A) Smear of peripheral blood. Prorubricytes (arrow) and basophilic rubricytes (arrowhead) were observed. Diff-Quick stain. (B) Smear of bone marrow aspiration specimen on day 8. Early-stage erythroid progenitors were prominent. Dysplastic erythroid cells with fragmentation of the nucleus (C: arrow) were observed. Diff-Quick stain.

either abnormal proliferation of erythroid progenitors with differentiation arrest or immune-mediated destruction of late-stage erythroid cells. Because myeloproliferative disorder is rare in FeLV-negative cats [9], the cat was treated with cyclosporine A ( $5 \mathrm{mg} / \mathrm{kg}$ q24h) to control immune-mediated destruction of late-stage erythroid cells. Serum iron was restored to a normal level by oral iron supplementation; however, the anemia was not improved by the treatment (Fig. 2). The numbers of erythroid progenitors with large, irregular nuclei were increased in peripheral blood. Plasma thymidine kinase activity was high $(636 \mathrm{IU} / l)$ compared with clinically healthy control cats $(<5 \mathrm{IU} / l: \mathrm{n}=22) 46$ days after the 1st examination, indicating a myeloproliferative condition, as shown in other species $[4,5,8]$. The cat was regarded as having an MDS-like condition and was treated with low-dose cytarabine (20 $\mathrm{mg} / \mathrm{m}^{2}$ q24h subcutaneously) with reference to treatment for human MDS patients $[7,13]$. The cat's hemogram improved after starting cytarabine therapy as evidenced by decreasing numbers of prorubricytes and basophilic rubricytes and an increasing number of late-stage erythroid cells (Fig. 2). However, chronic renal failure progressed just before the start of cytarabine treatment and was accompanied by anorexia, weakness and pleural effusion (transudate; specific gravity 1.030; containing erythroid progenitors). The cat died 51 days after the 1 st examination, and a necropsy was performed with permission from the cat's owner. Marked splenomegaly was macroscopically evident at necropsy. Histopathological examination revealed expansion of the red pulp occupied by a large number of early-stage erythroid cells (Fig. 3A). These cells were negative for CD3, CD79 $\alpha$, and Iba I antigen. A remarkable number of erythroid cells was also observed in vessels of other organs, including the liver, kidney and lung, indicating abnormal infiltration and proliferation of erythroid progenitor cells. Bone marrow was replaced by fibrinous connective tissue, and a small number of bone marrow hematopoietic cells were dispersed among fibrosing tissue. Fibers were moderately increased and appeared as a network of coarse fibers. With reference to the criteria applied by Bleu et al., this case might be classified as grade 2 myelofibrosis (moderate condition), though reticulin fibers were not examined in this case [1] (Fig. 3B). Proliferation of megakaryocytes, which is often seen in human patients with myelofibrosis, was not observed in this cat. Other tumors or significant findings that may have been associated with the cat's death were not found at necropsy.

The pathological condition of this cat is difficult to classify into a well-recognized hematological disease. The cat showed progressive anemia, and a low number of blasts (3.4\%/ANC) was present in the bone marrow. According to criteria in the World Health Organization classification for 


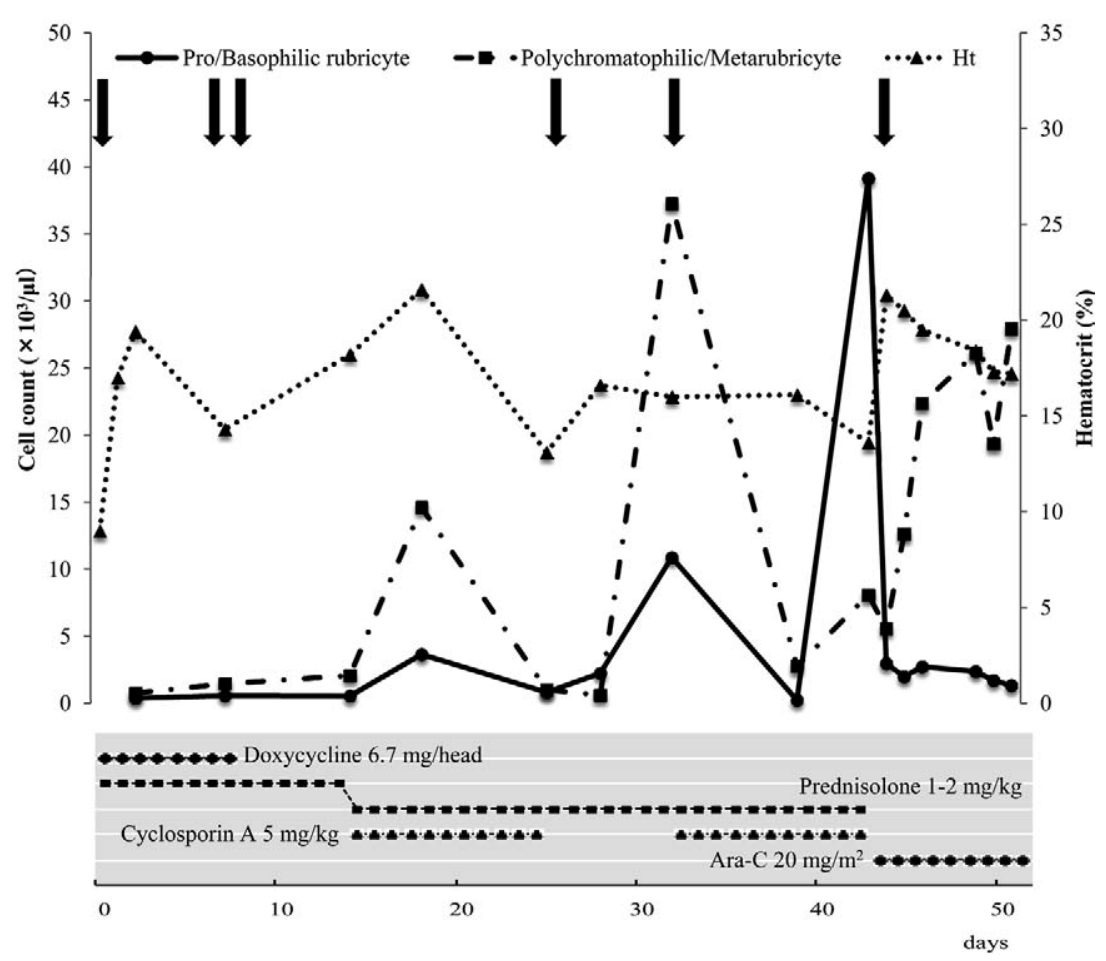

Fig. 2. Therapeutic time course and erythroid cell numbers during treatment. Arrows indicate days of blood transfusion. Erythroid cell counts at different mature stages and hematocrit value are shown. Reagents administered during the treatment period are also shown.

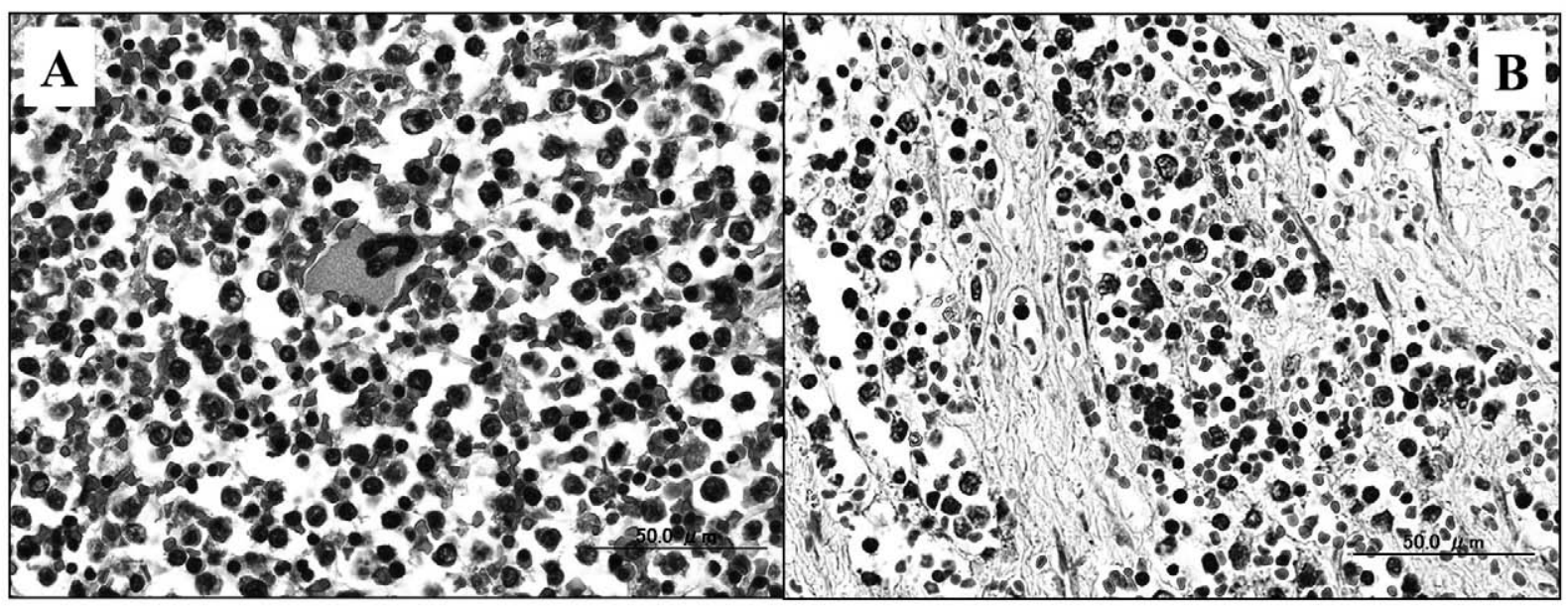

Fig. 3. (A) Histopathological specimen of the spleen obtained at necropsy. The spleen was occupied mostly by red pulp infiltrated with erythroid cells (pro/basophilic rubricytes). High magnification, HE stain. (B) Histopathological specimen of femoral bone marrow obtained at necropsy. Bone marrow was replaced by fibrous connective tissue, and a small number of bone marrow hematopoietic cells were dispersed among fibrosing tissue. High magnification, HE stain.

malignant hematological conditions [3, 12], this cat might be classified as MDS with refractory anemia (MDS-RA), though morphological dysplasia was minimal.

A characteristic finding of this case was erythroid hyperplasia with a predominance of early-stage progenitors. It is sometimes difficult to discriminate between myeloprolifera- tive disorder and immune-mediated erythroid depletion. Deceptive maturation arrest is reported in dogs with immunemediated destruction of erythroid precursor cells at a specific maturation stage [10]. However, evidence of an immune-mediated depletion, such as erythrophagocytosis or lymphoid aggregation, was not observed in this case. In patients with 
marked extramedullary erythropoiesis, early-stage erythroid cells often appear in peripheral blood. Because there were minimal dysplastic features in erythroid cells, extramedullary erythropoiesis secondary to myelofibrosis should be considered in this case. However, early-stage erythroid progenitors were predominant in extramedullary sites including pleural effusion, indicating uncontrolled proliferation of erythroid progenitor cells.

Myelofibrosis is rarely reported in cats. In a report of 44 cats with MDS or acute myelocytic leukemia, severe myelofibrosis, defined as an abundance of linear fibers distributed throughout the bone marrow, was observed in 5 cases [1]. Four of the 5 cats were positive for FeLV antigen. It is suspected that FeLV infection in stem cells can induce abnormal proliferation of hematopoietic cells, which eventually induces collagenous connective tissue fibers in bone marrow, as in myelofibrosis in humans. In this report, however, we have shown that a similar condition can occur in cats independently of FeLV infection. Although we treated the cat with cytarabine with reference to the treatment for humans, a standard treatment protocol for feline myeloproliferative disorder has not been proposed. Further understanding of the pathogenesis of feline myelofibrosis with an accumulation of cases may be helpful to establish an effective treatment.

\section{REFERENCES}

1. Blue, J. T. 1988. Myelofibrosis in cats with myelodysplastic syndrome and acute myelogenous leukemia. Vet. Pathol. 25: 154-160. [Medline] [CrossRef]

2. John, W. H. 2001. Hematopoiesis. pp. 87-91. In: Atlas of Veterinary Hematology., Saunders, Philadelphia.

3. McManus, P. M. 2005. Classification of myeloid neoplasms: a comparative review. Vet. Clin. Pathol. 34: 189-212. [Medline] [CrossRef]

4. Musto, P., Bodenizza, C., Falcone, A., D’Arena, G., Scalzulli, P., Perla, G., Modoni, S., Parlatore, L., Valvano, M. R. and Carotenuto, M. 1995. Prognostic relevance of serum thymidine kinase in primary myelodysplastic syndromes: relationship to development of acute myeloid leukaemia. Br. J. Haematol. 90: 125-130. [Medline] [CrossRef]

5. Nakamura, N., Momoi, Y., Watari, T., Yoshino, T., Tsujimoto, H. and Hasegawa, A. 1997. Plasma thymidine kinase activity in dogs with lymphoma and leukemia. J. Vet. Med. Sci. 59: 957960. [Medline] [CrossRef]

6. Nishigaki, K., Okuda, M., Endo, Y., Watari, T., Tsujimoto, H. and Hasegawa, A. 1997. Structure and function of the long terminal repeats of feline leukemia viruses derived from naturally occurring acute myeloid leukemias in cats. J. Virol. 71: 98239827. [Medline]

7. Powell, B. L., Capizzi, R. L., Jackson, D. V., Richards, F., Muss, H. B., Lyerly, E. S., Rosenbaum, D. L., Connelly, R. A., Buss, D. H., Bearden, J. D., et al. 1988. Low dose Ara-C for patients with myelodysplastic syndromes. Leukemia 2: 153-156. [Medline]

8. Sakamoto, L., Ohbayashi, T., Matsumoto, K., Kobayashi, Y. and Inokuma, H. 2009. Serum thymidine kinase activity as a useful marker for bovine leucosis. J. Vet. Diagn. Invest. 21: 871-874. [Medline] [CrossRef]

9. Shimoda, T., Shiranaga, N., Mashita, T. and Hasegawa, A. 2000. A hematological study on thirteen cats with myelodysplastic syndrome. J. Vet. Med. Sci. 62: 59-64. [Medline] [CrossRef]

10. Stokol, T., Blue, J. T. and French, T. W. 2000. Idiopathic pure red cell aplasia and nonregenerative immune-mediated anemia in dogs: 43 cases (1988-1999). J. Am. Vet. Med. Assoc. 216: 1429-1436. [Medline] [CrossRef]

11. Tanahara, M., Miyamoto, S., Nishio, T., Yoshii, Y., Sakuma, M., Sakata, Y., Nishigaki, K., Tsujimoto, H., Setoguchi, A. and Endo, Y. 2010. An epidemiological survey of feline hemoplasma infection in Japan. J. Vet. Med. Sci. 72: 1575-1581. [Medline] [CrossRef]

12. Vardiman, J. W., Harris, N. L. and Brunning, R. D. 2002. The World Organization (WHO) classification of the myeloid neoplasms. Blood 100: 2292-2302. [Medline] [CrossRef]

13. Wataya, S., Sudou, H., Sakamaki, I., Wano, Y., Ueda, T. and Nakamura, T. 1995. Successful treatment of a 74-year-old man with refractory anemia with excess of blasts in transformation (RAEB-T) by low-dose Ara-C injection. Nippon Ronen Igakkai Zasshi 32: 438-441. [Medline] [CrossRef] 